\title{
ON THE PRIME FACTORIZATION OF BINOMIAL COEFFICIENTS
}

\author{
E. F. ECKLUND, JR., R. B. EGGLETON, P. ERDÖS and J. L. SELFRIDGE
}

Dedicated to Kurt Mahler on his 75th birthday

(Received 18 January 1978)

Communicated by J. H. Coates

\begin{abstract}
For positive integers $n$ and $k$, with $n \geqslant 2 k$, let $\left(\begin{array}{l}n \\ k\end{array}\right)=u v$, where each prime factor of $u$ is less than $k$, and each prime factor of $v$ is at least equal to $k$. It is shown that $u<v$ holds with just 12 exceptions, which are determined. If $\left(\begin{array}{l}n \\ k\end{array}\right)=U V$, where each prime factor of $U$ is at most equal to $k$, and each prime factor of $V$ is greater than $k$, then $U<V$ holds with at most finitely many exceptions, 19 of which are determined. It is conjectured that there are no others.
\end{abstract}

Subject classification (Amer. Math. Soc. (MOS) 1970): 10A05, 10A25.

\section{Introduction}

In this paper our basic concern is with the product of the small prime factors in runs of consecutive integers. Let us fix a positive integer $k$ and examine runs of consecutive integers having no prime factor greater than $k$. Such runs cannot be very long (see Ecklund and Eggleton (1972)). Indeed, a theorem of Størmer (1897) shows there are only finitely many pairs of consecutive integers with no prime factor greater than $k$. Moreover, it was proved independently by Sylvester (1892) and Schur (1929) that any run of $k$ consecutive integers, each larger than $k$,

The second author's work was supported in part by the DeKalb Number Theory Foundation.

(C) Copyright Australian Mathematical Society 1978

Copyright. Apart from any fair dealing for scholarly purposes as permitted under the Copyright Act, no part of this JOURNAL may be reproduced by any process without written permission from the Treasurer of the Australian Mathematical Society. 
contains at least one multiple of a prime greater than $k$. This may be expressed as follows:

THEOREM (Sylvester-Schur). For positive integers $n$ and $k$, with $n \geqslant 2 k$, the binomial coefficient $\left(\begin{array}{l}n \\ k\end{array}\right)$ has a prime factor greater than $k$.

An elementary proof of the theorem in this form was given by Erdös (1934) and a proof of a stronger theorem, also essentially due to Erdös, appears in Ecklund and Eggleton (1972).

By a theorem of Mahler (1961), for any given real $\varepsilon>0$ and positive integer $k$, the largest divisor of $\left(\begin{array}{l}n \\ k\end{array}\right)$ consisting entitely of primes not exceeding $k$ is less than $n^{1+\epsilon}$, provided $n$ is sufficiently large. Note also that the largest power of 2 dividing $\left(\begin{array}{l}n \\ 2\end{array}\right)$ cannot exceed $\frac{1}{2} n$. Thus with Mahler's Theorem we deduce the following result, which contains more quantitative information than the Sylvester-Schur Theorem, though it lacks an effective bound on $k$.

THEOREM. For positive integers $n$ and $k$, let $\left(\begin{array}{l}n \\ k\end{array}\right)=U V$, where the prime factors of $U$ do not exceed $k$ and the prime factors of $V$ are all greater than $k$. Then $U<V$ provided $n$ is sufficiently large compared with $k$.

Of course $U$ and $V$ depend on $n$ and $k$ in this theorem, but it is convenient not to make this explicit in the notation.

When discussing the prime factors of runs of $k$ consecutive integers, it is in fact natural to distinguish between primes which could possibly divide two or more members of the run, and those which are larger so can divide at most one member of the run: in other words, to distinguish primes strictly less than $k$ from those at least as large as $k$. In this paper our main theme is the proof of the following fact.

THEOREM. For positive integers $n$ and $k$, with $n \geqslant 2 k$, let $\left(\begin{array}{l}n \\ k\end{array}\right)=u v$, where the prime factors of $u$ are all less than $k$ and the prime factors of $v$ are all at least as large as $k$. Then $u>v$ holds in just 12 cases, namely

$$
\left(\begin{array}{l}
8 \\
3
\end{array}\right),\left(\begin{array}{l}
9 \\
4
\end{array}\right),\left(\begin{array}{c}
10 \\
5
\end{array}\right),\left(\begin{array}{c}
12 \\
5
\end{array}\right),\left(\begin{array}{c}
21 \\
7
\end{array}\right),\left(\begin{array}{c}
21 \\
8
\end{array}\right),\left(\begin{array}{c}
30 \\
7
\end{array}\right),\left(\begin{array}{l}
33 \\
13
\end{array}\right),\left(\begin{array}{l}
33 \\
14
\end{array}\right),\left(\begin{array}{l}
36 \\
13
\end{array}\right),\left(\begin{array}{l}
36 \\
17
\end{array}\right),\left(\begin{array}{l}
56 \\
13
\end{array}\right) .
$$


Using the notation $\left(\begin{array}{l}n \\ k\end{array}\right)=U V$, as in the earlier theorem, we shall show as a sorollary to part of the proof of the above theorem that there are only finitely many cases with $n \geqslant 2 k$ for which $U>V$. Seven cases where this occurs, in addition to the 12 with $u>v$, are the following:

$$
\left(\begin{array}{l}
9 \\
3
\end{array}\right),\left(\begin{array}{c}
10 \\
3
\end{array}\right),\left(\begin{array}{c}
18 \\
3
\end{array}\right),\left(\begin{array}{c}
28 \\
5
\end{array}\right),\left(\begin{array}{c}
54 \\
7
\end{array}\right),\left(\begin{array}{c}
82 \\
3
\end{array}\right),\left(\begin{array}{c}
162 \\
3
\end{array}\right) .
$$

In addition, we note that $\left(\begin{array}{c}514 \\ 3\end{array}\right)$ is a near miss, with $V / U<1.06$.) However, Mahler's Theorem does not give an effective upper bound on the solutions, and we are unable to prove completeness of our list for cases with $k=3,5,7$, though $t$ is complete for all other values of $k$. We strongly conjecture that the list is also :omplete for these three problematic values of $k$.

\section{Plan of attack}

For convenience, we shall frequently replace $n$ by $c k$, where $c \geqslant 2$ is a rational rariable such that $c k$ is always an integer. We wish to show that if $\left(\begin{array}{c}c k \\ k\end{array}\right)=u v$, where the product separates prime factors less than $k$ from those greater than or :qual to $k$, then $u>v$ holds in only 12 cases. To do this we divide the problem into ive distinct parts, represented in Diagram 1.

In Region I, where $k$ and $c$ are both large, we show that $\left(\begin{array}{c}c k \\ k\end{array}\right)>u^{2}$ by comparing he binomial coefficient with the square of a simple overestimate for the product of ts small prime factors. In Region II, where $k$ is large and $c$ is small, we show that $\left.\begin{array}{l}c k \\ k\end{array}\right)<v^{2}$ by comparing the binomial coefficient with the square of an understimate for the product of its large prime factors. In Region III, where $k$ is small ind $c$ is large, we need to make a careful overestimate of $u$ and compare it with the :orresponding underestimate of $v$, showing that $c$ is large enough for $v$ to dominate. n Region IV, where $k$ and $c$ are both relatively small, all cases are directly examined y computer. This checking is carried out for each $k$ in the range $1 \leqslant k \leqslant 494$. For ertain $k \leqslant 24$ it turns out that the lower bound on $c$ (which we calculate to ensure hat $u<v$ ) lies above the top of the search range for Region IV, obtained by extrapoation of the lower bound used for Region III. Region V comprises these remaining ases, which we finally eliminate by more sensitive systematic estimates of the size if $u$. In fact, to get the upper bound for Region $V$ in three cases, we reduce the 
possible instances to occurrences of special configurations of numbers with no large prime factors, and use the tabulation due to Lehmer (1964) to locate and examine all such configurations.

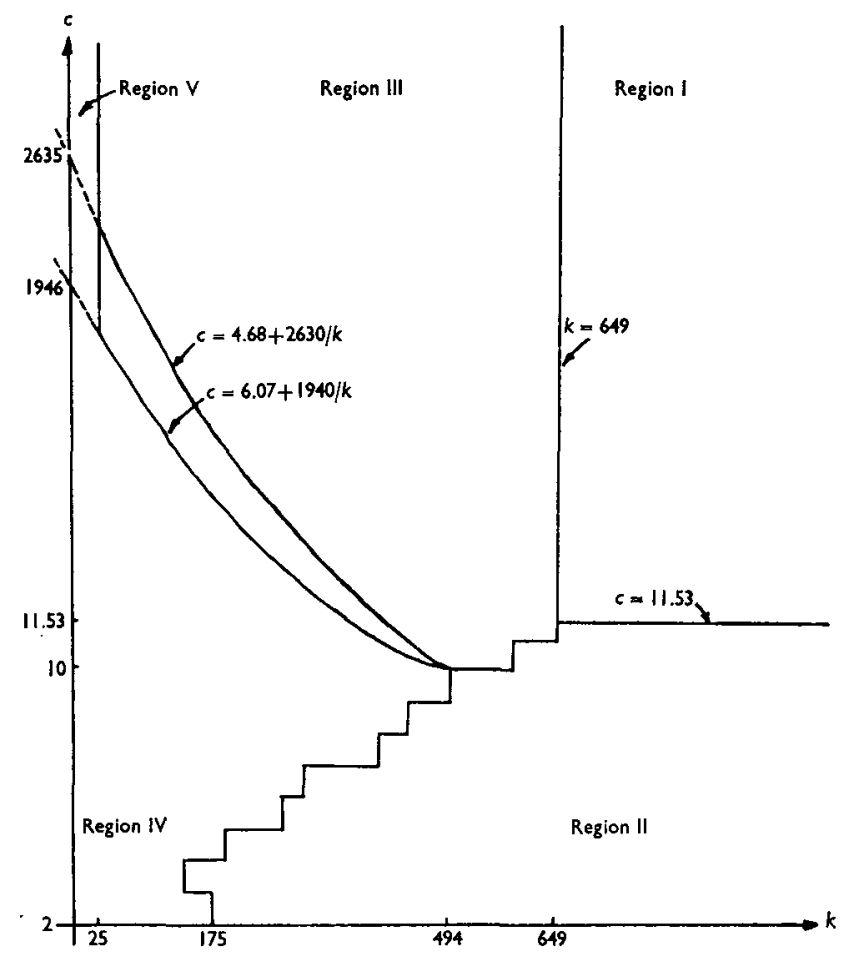

Diagram 1. The regions for the various arguments used in the proof.

\section{Region I: $k$ and $c$ both large}

A basic estimate, given in Erdös (1934) and Erdös and Graham (1976), shows that if $p^{\alpha}$ is a divisor of $\left(\begin{array}{l}n \\ k\end{array}\right)$, then $p^{\alpha} \leqslant n$. Hence

$$
u \leqslant n^{\pi(k-1)},
$$

where $\pi(x)$ denotes the number of primes not exceeding $x$. By a result of Rosser and Schoenfeld (1975), we have

$$
\pi(x)<\left(1+\frac{3}{2 \log x}\right) \frac{x}{\log x} \text { for } x>1 .
$$

Thus, if we anticipate the bound on $k$ for Region I and take $k \geqslant 649$ and $n=c k$, it follows that

$$
\log u<1.23165 k \log c k / \log k
$$


To get a suitable bound on the binomial coefficient $\left(\begin{array}{c}c k \\ k\end{array}\right)$, we use Stirling's formula,

$$
n !=\sqrt{ }(2 \pi n)\left(\frac{n}{e}\right)^{n} e^{\delta / 12 n} \text { for } n \geqslant 1,
$$

where $\delta$ is a real number depending on $n$, and satisfying $0<\delta<1$. With $k \geqslant 649$ and $c \geqslant 11.53$, it follows from (4) that

$$
\frac{1}{k} \log \left(\begin{array}{c}
c k \\
k
\end{array}\right)>c \log c-(c-1) \log (c-1)-0.00641 \text {. }
$$

The desired inequality is $u<v$, which is equivalent to

$$
\left(\begin{array}{c}
c k \\
k
\end{array}\right)>u^{2}
$$

By (3) and (5), this certainly holds if

$$
c \log c-(c-1) \log (c-1)-0.00641>2.46330(1+\log c / \log k) .
$$

I routine calculation with $k \geqslant 649$ verifies that ( 7$)$ holds for $c \geqslant 11.53$, so it follows hat $u<v$ (and indeed $U<V$ ) holds in the region determined by these bounds on $c$ and $c$. (Of course, we arrived at these particular bounds on $k$ and $c$ for Region $I$ sy successive approximation, with an eye to the bounds forced on us by our nethods for dealing with Regions II and III. If we reduced the bound on $c$ in Region I, it would be at the expense of increasing the bound on $k$.)

\section{Region II: $k$ large, $c$ small}

With $\left(\begin{array}{c}c k \\ k\end{array}\right)=u v$, the definition of $v$ ensures that it is divisible by every prime retween $(c-1) k$ and $c k$, for any $c \geqslant 2$. Indeed, for any positive integer $r \leqslant c$, we ee that $v$ must be divisible by each prime which is between $(c-1) k / r$ and $c k / r$ ind which is at least as large as $k$. Let $P_{r}$ denote the product of the set of primes $p$ atisfying $(c-1) k / r<p \leqslant c k / r$ and $p \geqslant k$, for any positive integer $r \leqslant c$. Then we have

$$
v \geqslant \prod_{r \leqslant c} P_{r}
$$

1 recent result of Schoenfeld, reported in a footnote added in proof in Schoenfeld 1976), gives a sharp upper bound on $\theta(x)$, which is the sum of the logarithms of ll primes not exceeding $x$. This bound is

$$
\theta(x)=\sum_{p \leqslant x} \log p \leqslant 1.000081 x \text { for } x \geqslant 1 .
$$

n order to estimate the product $P_{r}$ we also need lower bounds on $\theta(x)$ for values 
of $x$ up to about $10^{4}$. Write

$$
\theta(x)=\sum_{p \leqslant x} \log p \geqslant \alpha x .
$$

A short table of values of $\alpha$, and associated lower bounds on $x$, is given by Rosser and Schoenfeld (1962) and supplemented in Rosser and Schoenfeld (1975), where the bound

$$
\theta(x) \geqslant 0.990 x \text { for } x \geqslant 32057
$$

is given. The best value of $\alpha$ available, when a lower bound on $x$ is given, can be deduced from these bounds of Rosser and Schoenfeld in conjunction with the tabulations of Appel and Rosser (1961). However, since the latter are relatively inaccessible, we present a table of values of $\alpha$ when the lower bound on $x$ lies in the interval up to 32057 (see Table 1). This table is based on direct computation

TABle 1

\begin{tabular}{|c|c|c|c|c|c|c|c|}
\hline$x_{0}$ & $\alpha$ & $x_{0}$ & $\alpha$ & $x_{0}$ & $\alpha$ & $x_{0}$ & $\alpha$ \\
\hline 2 & 0.231 & 593 & 0.9291 & 5381 & 0.97526 & 14387 & 0.98551 \\
\hline 3 & 0.358 & 599 & 0.9367 & 5387 & 0.97577 & 14401 & 0.98576 \\
\hline 5 & 0.485 & 601 & 0.9380 & 5393 & 0.97628 & 14407 & 0.98578 \\
\hline 7 & 0.486 & 607 & 0.9383 & 5399 & 0.97642 & 14533 & 0.98608 \\
\hline 11 & 0.595 & 809 & 0.9409 & 5407 & 0.97693 & 19373 & 0.98628 \\
\hline 13 & 0.606 & 821 & 0.9449 & 5413 & 0.97749 & 19379 & 0.98669 \\
\hline 17 & 0.662 & 853 & 0.9455 & 5639 & 0.97867 & 19381 & 0.9868973 \\
\hline 29 & 0.703 & 1423 & 0.9480 & 5641 & 0.97886 & 19387 & 0.9868979 \\
\hline 37 & 0.722 & 1427 & 0.9517 & 7451 & 0.97903 & 19417 & 0.98720 \\
\hline 41 & 0.761 & 1429 & 0.9541 & 7477 & 0.97970 & 19421 & 0.98760 \\
\hline 59 & 0.792 & 1433 & 0.9550 & 7481 & 0.98011 & 19423 & 0.98791 \\
\hline 67 & 0.807 & 1447 & 0.9573 & 7487 & 0.98092 & 19427 & 0.98821 \\
\hline 71 & 0.816 & 1451 & 0.9576 & 7499 & 0.98094 & 19681 & 0.9884167 \\
\hline 97 & 0.828 & 1481 & 0.9600 & 7517 & 0.98110 & 19687 & 0.9884169 \\
\hline 101 & 0.843 & 1973 & 0.9609 & 8597 & 0.98129 & 19697 & 0.98862 \\
\hline 127 & 0.8499 & 1987 & 0.9618 & 8623 & 0.98189 & 19913 & 0.98872 \\
\hline 149 & 0.8694 & 1993 & 0.9629 & 8627 & 0.98199 & 19961 & 0.98878 \\
\hline 163 & 0.8695 & 2237 & 0.9632 & 8663 & 0.98228 & 20873 & 0.98897 \\
\hline 223 & 0.8780 & 2657 & 0.9654 & 11777 & 0.98291 & 20879 & 0.989074 \\
\hline 227 & 0.8940 & 2659 & 0.9669 & 11779 & 0.98337 & 20887 & 0.989077 \\
\hline 229 & 0.8980 & 3299 & 0.9688 & 11783 & 0.98346 & 20897 & 0.989080 \\
\hline 347 & 0.9096 & 3301 & 0.96952 & 11801 & 0.98376 & 21481 & 0.989268 \\
\hline 349 & 0.9130 & 3307 & 0.96962 & 11807 & 0.98405 & 21487 & 0.989548 \\
\hline 367 & 0.9134 & 3449 & 0.96973 & 11813 & 0.98418 & 21491 & 0.989835 \\
\hline 419 & 0.9160 & 3457 & 0.97097 & 11821 & 0.98420 & 31957 & 0.989845 \\
\hline 431 & 0.9194 & 3461 & 0.97107 & 11897 & 0.98441 & 32051 & 0.989984 \\
\hline 557 & 0.9208 & 3511 & 0.97130 & 11923 & 0.98487 & 32057 & 0.990 \\
\hline 563 & 0.9222 & 3527 & 0.97306 & 11927 & 0.98500 & & \\
\hline 569 & 0.9264 & 3529 & 0.97427 & 12097 & 0.98509 & & \\
\hline 587 & 0.9278 & 3533 & 0.97475 & 12373 & 0.98513 & & \\
\hline
\end{tabular}

Values of $x_{0}$ and successive infima for $\alpha$ such that $\theta(x) \geqslant \alpha x$ for all real $x \geqslant x_{0}$ 
of $\theta(x) / x$ for the primes up to 32057 , and uses (11) to cover the region beyond this point.

Recall that the desired inequality is $u<v$, which is equivalent to

$$
\left(\begin{array}{c}
c k \\
k
\end{array}\right)<v^{2}
$$

From (4) we have

$$
\frac{1}{k} \log \left(\begin{array}{c}
c k \\
k
\end{array}\right)<c \log c-(c-1) \log (c-1) \text { for } c>1 \text {. }
$$

Now, using (8) and (13), if $m \leqslant c<m+1$ for some integer $m \geqslant 2$, the inequality (12) is certainly satisfied if

$$
\frac{1}{2}\left(\log (m+1)-\frac{m}{m+1} \log m\right)+\frac{\beta m}{m+1} \sum_{r \leqslant m} \frac{1}{r}<\sum_{r \leqslant m} \frac{\alpha(r)}{r},
$$

where $\beta=1.000081$ comes from (9), and $\alpha(r)$ is the value of $\alpha$ in (10) which holds for $x \geqslant m k / r$. By successive approximation using Table 1 , we obtain a lower bound on the value of $k$ for which (12) certainly holds when $m \leqslant c<m+1$. This information, for $2 \leqslant m \leqslant 11$, is given in Table 2 . The left boundary of Region II is determined by this data (see Diagram 1). Thus (12) is established over a range of $c$ which reaches (and overlaps) the range covered by Region I. The method clearly establishes $U<V$ at the same time.

TABLE 2

Values of $m$ and $k_{0}(m)$ such that inequality (14) is satisfied if $k \geqslant k_{0}(m)$

\begin{tabular}{ccrrr}
\hline$m$ & $k_{0}(m)$ & & $m$ & $k_{0}(m)$ \\
\cline { 1 - 2 } \cline { 4 - 5 } 2 & 175 & & 7 & 398 \\
3 & 153 & & 8 & 433 \\
4 & 206 & & 9 & 494 \\
5 & 278 & & 10 & 571 \\
6 & 300 & & 11 & 649 \\
\hline
\end{tabular}

\section{Region III: $k$ small, $c$ large}

By expressing $\left(\begin{array}{l}n \\ k\end{array}\right)$ in the form $u v=n(n-1) \ldots(n-k+1) / k$ ! a good overestimate for $u$ can be obtained as follows. For any prime $p<k$, let $\lambda(p)$ be the maximum exponent of the powers of $p$ occurring as factors of any of the integers $n, n-1, \ldots, n-k+1$. Thus

$$
\lambda(p)=\max \left\{\mu(a): p^{\mu(x)} \| a, \quad n \geqslant a>n-\dot{k}\right\} .
$$


Also let $a_{p}$ be the corresponding largest multiple of $p^{\lambda(p)}$, that is,

$$
a_{p}=\max \left\{a: p^{\lambda(p)} \mid a, \quad n \geqslant a>n-k\right\} .
$$

We consider the set of these multiples of maximum powers of small primes,

$$
S(n, k)=\left\{a_{p}: p<k\right\}
$$

where the cardinality of $S(n, k)$ is at most $\pi(k-1)$, and may be less since it is possible that $a_{p}=a_{q}$ occurs for distinct primes $p, q$.

For any prime $p<k$, we define the intrinsic exponent $\kappa(p, n, k)$ of $p$ in the product $n(n-1) \ldots(n-k+1)$ to be the maximum exponent $\kappa$ for which $p^{\kappa}$ is a factor of $n(n-1) \ldots(n-k+1) / a_{p}$. Note that if $n-a_{p} \geqslant i>n-k-a_{p}$ and $i \neq 0$, then $p^{\mu} \| a_{p}+i$ implies $p^{\mu} \| i$, since no $i$ can contain $p$ to a higher power than $\lambda(p)$. Thus $\kappa(p, n, k)$ is equal to the maximum exponent for which $p^{x}$ divides the product $\left(n-a_{p}\right) !\left(a_{p}-n+k-1\right)$ ! and this product divides $(k-1) !$ since $\left(\begin{array}{c}k-1 \\ n-a_{p}\end{array}\right)$ is an integer. Now let $P(n, k)$ denote the intrinsic part of the product $n(n-1) \ldots(n-k+1)$, defined by

$$
P(n, k)=\prod_{p<k} p^{\kappa(p, n, k)} .
$$

Then we have just shown that

$$
P(n, k) \mid(k-1) !
$$

If $k$ is composite, all prime factors of $k$ ! are less than $k$, so $u \leqslant P(n, k) \Pi(S) / k$ !, where $\Pi(S)$ is the product of the integers in $S(n, k)$. Taking the largest possible elements for $S(n, k)$, and the greatest possible number, and using (19) to provide the bound $P(n, k) \leqslant(k-1)$ !, we get

$$
u \leqslant n(n-1) \ldots(n-\pi+1) / k \text { for } k \text { composite, }
$$

where $\pi=\pi(k-1)$. Similarly, if $k$ is prime, the product of prime factors of $k$ ! which are less than $k$ is $(k-1)$ !, and the corresponding estimates lead to

$$
u \leqslant n(n-1) \ldots(n-\pi+1) \text { for } k \text { prime, }
$$

where $\pi=\pi(k-1)$ as before.

It is now clear from (20) and $\left(20^{\prime}\right)$ that the desired inequality $u<v$ follows if

$$
(k-1) ! n(n-1) \ldots(n-\pi+1)<k(n-\pi)(n-\pi-1) \ldots(n-k+1)
$$

and if

for $k$ composite,

(21') $k ! n(n-1) \ldots(n-\pi+1)<(n-\pi)(n-\pi-1) \ldots(n-k+1)$ for $k$ prime. 
If $k>2 \pi(k-1)$, the left members of (21) and $\left(21^{\prime}\right)$ are of lower degree in $n$ than the right members: this actually holds for all $k$ except $k=4,6$ and 8 . So for each $k$, apart from these three exceptions, we can determine the smallest value of $n=c k$ such that the corresponding one of (21) and $\left(21^{\prime}\right)$ holds. To deal with cases not covered in Regions I and II, we computed this smallest $n$ for $k \leqslant 649$, and for simplicity determined the following linear bound from our data, so (21) and (21') hold if

$$
n=c k \geqslant 6.07 k+1940 \text { for } 25 \leqslant k \leqslant 649 .
$$

This determines the boundary of Region III. For $k \leqslant 25$, the corresponding lower bounds are given in Table 3. Apart from the three cases in which the method does not apply, it is evident that (22) is actually a justified bound except when $k=7,9$, $14,18,19,20,21$ and 24.

TABLE 3

Values of $k$ and $n_{1}(k)$ such that inequalities (21) and (21') are satisfied if $n \geqslant n_{1}(k)$

\begin{tabular}{|c|c|c|c|c|c|}
\hline$k$ & $n_{1}(k)$ & $k$ & $n_{1}(k)$ & $k$ & $n_{1}(k)$ \\
\hline 2 & 3 & 10 & 207 & 18 & 2137 \\
\hline 3 & 9 & 11 & 356 & 19 & 2639 \\
\hline 4 & - & 12 & 1847 & 20 & 8865 \\
\hline 5 & 128 & 13 & 1860 & 21 & 2618 \\
\hline 6 & - & 14 & 21121 & 22 & 1180 \\
\hline 7 & 5055 & 15 & 1823 & 23 & 1620 \\
\hline 8 & - & 16 & 557 & 24 & 3236 \\
\hline 9 & 4504 & 17 & 835 & 25 & 1615 \\
\hline
\end{tabular}

As indicated in the Introduction, we are also interested in determining all instances of $\left(\begin{array}{l}n \\ k\end{array}\right)$ with $n \geqslant 2 k$ for which $U>V$. When $k$ is composite, these are just the instances for which $u>v$. When $k$ is prime, $\left(20^{\prime}\right)$ is replaced by

$$
U \leqslant n(n-1) \ldots(n-\pi) / k \text { for } k \text { prime, }
$$

where $\pi=\pi(k-1)$ as before. We can ensure that $U<V$ by requiring (21") $\quad(k-1) ! n(n-1) \ldots(n-\pi)<k(n-\pi-1) \ldots(n-k+1)$ for $k$ prime.

The linear bound

$$
n=c k \geqslant 4.68 k+2630 \text { for } 25 \leqslant k \leqslant 649
$$

corresponds to the bound (22), and ensures that $\left(21^{\prime \prime}\right)$ holds. The left member of $\left(21^{\prime \prime}\right)$ is of lower degree in $n$ than the right member for every prime $k>7$. So apart from $k=3,5$ and 7 (where our methods do not yield an explicit bound), the lower bounds on $n$ for validity of $\left(21^{\prime \prime}\right)$ for odd prime $k \leqslant 23$ are given in Table 4. 
TABLE 4

Values of $k$ and $n_{2}(k)$ such that inequality (21")

is satisfied if $n \geqslant n_{2}(k)$

\begin{tabular}{rcrrr}
\hline$k$ & $n_{2}(k)$ & & $k$ & \multicolumn{1}{c}{$n_{2}(k)$} \\
\cline { 1 - 2 } \cline { 5 - 5 } 5 & - & & 13 & 36846325 \\
5 & - & & 17 & 10748 \\
7 & - & & 19 & 69626 \\
11 & 329926 & & 23 & 8702 \\
\hline
\end{tabular}

\section{Region IV: $k$ and $c$ both small}

To investigate the Region IV, where $k \geqslant 1$ is subject to the upper bounds in Table 2, and $c \geqslant 2$ is subject to the upper bound (22), a simple computer-assisted search was carried out. In practice, for $c$ we used the bound (22'), so that instances for which $U>V$ holds were also determined. All the instances listed in the Introduction were found this way. (Indeed, the near miss $\left(\begin{array}{c}514 \\ 3\end{array}\right)$ is the only other instance in the region with $V / U<1.1)$.

\section{Region $\mathrm{V}: k \leqslant 24, c$ large}

Here we sharpen the techniques applied to Region III. The intrinsic part $P(n, k)$ of the product $n(n-1) \ldots(n-k+1)$ was defined in (18). We now also define the extrinsic part $Q(n, k)$ of this product, by

$$
Q(n, k)=\Pi(S) / \prod_{p<k} p^{\lambda(p)},
$$

where $\Pi(S)$ is the product of all the integers in $S(n, k)$, given in (17). Thus $Q(n, k)$ is the product of prime factors greater than or equal to $k$ in the numbers $a_{p}$. With $\pi=\pi(k-1)$, we can now write

$$
u \leqslant \frac{n(n-1) \ldots(n-\pi+1)}{k !} \cdot \frac{P(n, k)}{Q(n, k)} \text { for } k \text { composite, }
$$

and $k$ times this bound for $k$ prime. Since $\left(\begin{array}{l}n \\ k\end{array}\right)=u v$, the desired inequality $u<v$ certainly holds if

$$
n(n-1) \ldots(n-\pi+1)<(n-\pi)(n-\pi-1) \ldots(n-k+1) \cdot R(n, k),
$$


where

$$
R(n, k)= \begin{cases}k !\left(\frac{Q(n, k)}{P(n, k)}\right)^{2} & \text { for } k \text { composite } \\ \frac{(k-1) !\left(\frac{Q(n, k)}{P(n, k)}\right)^{2}}{k} & \text { for } k \text { prime. }\end{cases}
$$

We use (25) to deal with the troublesome cases $\left(\begin{array}{l}n \\ k\end{array}\right)=u v$ with $k=4,6$ and 8 . To illustrate the method, the case $k=8$ will now be discussed briefly.

Using the notation introduced in (17), if $|S(n, 8)| \leqslant 3$ it is easy to verify that $u<v$ must hold if $n \geqslant 36$. So now suppose $|S(n, 8)|=4$. If $P(n, 8)^{2}<8$ !, it follows from (19) that $P(n, 8) \leqslant 180$, and then $R(n, 8) \geqslant 56 / 45$, using (26). In this case, (25) holds if $n \geqslant 77$. For larger $P(n, 8)$ we still have $P(n, 8) \leqslant 7$ ! by $(19)$, so if $Q(n, 8) \geqslant 29$ then $R(n, 8) \geqslant 841 / 630>56 / 45$, so (25) certainly holds if $n \geqslant 77$. It remains to check the cases with $Q(n, 8)<29$. By (23), the only possibilities are

$$
Q(n, 8) \in\{1,11,13,17,19,23\} \text {. }
$$

Moreover, the direct search reported in the previous section was carried out up to $n=2667$ for $k=8$, according to $\left(22^{\prime}\right)$. Thus it remains to locate all those runs of $k=8$ consecutive integers, with largest member $n \geqslant 2668$, which contain three numbers having no prime factor greater than 7 , and a fourth with at most one prime factor (counting multiplicity) greater than 7 , but none greater than 23 . Either the first three contain a pair of the form $a, a+d$ with $d=1,2$ or 4 , or else the first three are of the form $a, a+3, a+6$, in which case the fourth is necessarily adjacent to one of them. All occurrences of such configurations can be deduced from the tables in Lehmer (1964), by first locating all possible pairs described. Each potential configuration is easily tested and rejected, so no further instances of $u>v$ with $k=8$ exist.

The other cases to be checked for $u>v$ are $k=7,9,14,20$ and 24, and those to be checked for $U>V$ are $k=11,13,17,19$ and 23. Tables 3 and 4 give the upper bound on $n$ for each case, while (22') gives the lower bound. Again we illustrate the method by brief discussion of one case: we choose $k=14$ for this purpose.

Let $A(n, 14)$ denote the product, running over each prime $p<14$, of the largest prime-powers $p^{\alpha} \leqslant n$. Combining this with (18) and (19), we observe that

$$
u \leqslant A(n, 14) P(n, 14) / 14 ! \leqslant A(n, 14) / 14 \text {. }
$$

Table 3 gives the upper bound $n \leqslant 21120$, and $A(21120,14)=2^{14} 3^{9} 5^{6} 7^{5} 11^{4} 13^{3}$. Correspondingly we have $v \geqslant(n-6)(n-7) \ldots(n-13) / 13$ ! so $u<v$ holds provided 
$n \geqslant 13669$. Iterating the calculation with this new bound,

$$
A(13668,14)=2^{13} 3^{8} 5^{5} 7^{4} 11^{3} 13^{3}
$$

shows that $u<v$ holds provided $n \geqslant 5198$. A further iteration leads only to $n \geqslant 4157$, and $A(5197,14)=A(4156,14)$. However, we can get down to the lower bound $n \geqslant 2695$ coming from $\left(22^{\prime}\right)$ by noting that $P(n, 14) \equiv 0(\bmod 13)$ holds only if $n \equiv 0(\bmod 13)$. Thus, for $n \leqslant 4156$ we have either the bound $u \leqslant A(4156,14) / 13.14$, which is sharper than (27) by a factor of 13, or else one of $n$ and $n-13$ is a multiple of 2197 , the largest available power of 13 . In this example, observe that there is in fact no multiple of 2197 between the current search bounds. The sharper bound on $u$ ensures that $u<v$ holds throughout the current search range, so the checking is complete. (We also made a separate check using more intricate combinatorial arguments, in conjunction with Lehmer's tables, for all the relevant cases in Region V.)

\section{Remarks and unsolved problems}

Here we shall use notation which makes explicit the dependence of $U$ and $V$ on $n$ and $k$, where as usual we have $n \geqslant 2 k$.

The most obvious outstanding problem is to obtain an effective upper bound on $n$ for which $U(n, k)>V(n, k)$ when $k=3,5$ or 7 . More generally, note that Mahler's Theorem that $U(n, k)<n^{1+\varepsilon}$ is not effective. It would be very interesting to obtain an effective result of the same kind, even if the result in question were much weaker. For example, it would be useful to have $U(n, k)<n^{k / 2}$ for $k>k_{0}$, with an explicit $k_{0}$.

An inequality of the form $U(n, k)<n^{2} e^{k}$, which may hold for $n<e^{k}$, would be useful. Perhaps such an inequality even holds if $n^{2}$ is replaced by $n$.

It would be of interest to strengthen Mahler's Theorem. For fixed $k$, perhaps there are positive constants $c_{1}$ and $c_{2}$ such that we have $U(n, k)<c_{1} n(\log n)^{c_{2}}$, for all sufficiently large $n$.

Consider, for fixed $k$, the sequence of integers $n(k, r)$ with $r=1,2, \ldots$, defined by taking $n(k, 1)=2 k$ and thereafter

$$
n(k, r+1)=\min \{n>n(k, r): U(n, k)>U(n(k, r), k)\} .
$$

It would be interesting to study the properties of this sequence, which is analogous to Ramanujan's sequence of highly composite numbers. Also of interest would be the properties of the strictly increasing sequence $N(k, r)$ with $r=1,2, \ldots$, where $N(k, r)$ is the $r$ th positive integer for which there is some constant $c(k, r)>1$ such that $U(n, k) / n^{c(k, r)}$ achieves its maximum at $n=N(k, r)$. This sequence is analogous to Ramanujan's sequence of superior highly composite numbers.

In closing, we mention that other results closely related to the present paper are given in Erdös and Graham (1976). 


\section{References}

K. I. Appel and J. Barkley Rosser (1961), Table for estimating functions of primes, Communications Research Division Tech. Report 4, Inst. for Defence Analyses, Princeton, N.J.

E. F. Ecklund, Jr., and R. B. Eggleton (1972), "Prime factors of consecutive integers", Amer. Math. Monthly 79, 1082-1089.

P. Erdös (1934), “A theorem of Sylvester and Schur”, J. London Math. Soc. 9, 282-288.

P. Erdös and R. L. Graham (1976), "On the prime factors of $\left(\begin{array}{l}n \\ k\end{array}\right)$ ", Fibonacci Quart. 14, 348-352.

D. H. Lehmer (1964), "On a problem of Størmer", Illinois J. Math. 8, 57-79.

K. Mahler (1961), Lectures on Diophantine Approximations I, University of Notre Dame. See Theorem (5, II), p. 159. (This is the most accessible reference, though not the earliest.)

J. Barkley Rosser and L. Schoenfeld (1962), "Approximate formulas for some functions of prime numbers", Illinois J. Math. 6, 64-94.

J. Barkley Rosser and L. Schoenfeld (1975), "Sharper bounds for the Chebyshev functions $\theta(x)$ and $\psi(x)$ ", Math. Comp. 29, 243-269.

L. Schoenfeld (1976), "Sharper bounds for the Chebyshev functions $\theta(x)$ and $\psi(x), I$ ", Math. Comp. 30, 337-360.

I. Schur (1929), "Einige Sätze über Primzahlen mit Anwendungen auf Irreduzibilitätsfragen", S.B. Deutsch. Akad. Wiss. Berlin Kl. Math. Phys. Tech. 23, 1-24.

G. Størmer (1897), Quelques Théorèmes sur l'Equation de Pell $x^{2}-D y^{2}= \pm 1$ et leurs Applications, Videnskabs-Selskabets Skrifter, Christiania, 2, $48 \mathrm{pp}$.

J. J. Sylvester (1892), “On arithmetical series”, Messenger Math. 21, 1-19, 87-120. (Collected Mathematical Papers, vol. 4, 1912, 687-731.)

Department of Computer Science

Oregon State University

Corvallis, Oregon 97330

USA

Hungarian Academy of Sciences

Reáltanoda U. 13-15

Budapest V

Hungary
Department of Mathematics

The University of Newcastle

New South Wales 2308

Australia

Department of Mathematical Sciences

Northern Illinois University

DeKalb, Illinois 60115

USA 